\title{
Rosetta awakes
}

\author{
ESA's Rosetta spacecraft has begun the next phase of its ambitious mission to land a probe on the \\ nucleus of a comet, and ride with the comet towards the Sun.
}

String and sealing wax - although probably no longer physicists' preferred ingredients for their experiments, these laboratory staples now rather represent an approach to scientific exploration. Plenty of progress in modern physics is made through tweaking and tinkering, the quick fix and the gap plugged. But there are many physicists for whom an improvised patch-up is not an option: the Large Hadron Collider, for example, affords its experimenters only limited windows of opportunity for repairs or upgrades. However, it is astronomers who, with perhaps only a single shot at their goal, are under the most pressure to get it right first time.

As this issue of Nature Physics went to press, astronomers were experiencing one of those knife-edge moments that characterize so many missions in space. After three years of hibernation out beyond Jupiter, the Rosetta spacecraft has been woken up: its alarm call - sent from ESA's control room in Darmstadt, Germany kicked off a nail-biting process of warm-up and reorientation. Success was confirmed only several hours later, when a signal from Rosetta was received at NASA's 70-m dish in Goldstone, California.

Rosetta launched in 2004 and has executed a series of slingshot manoeuvres, plus its period of no-solar-power hibernation, that put it on course to meet the comet $67 \mathrm{P} /$ Churyumov-Gerasimenko later this year. It has already completed a successful fly-by of two asteroids: a seven-minute encounter at a distance of $800 \mathrm{~km}$ with the small Šteins in 2008 and, in 2010, an approach to within $3,200 \mathrm{~km}$ of Lutetia, the then largest asteroid ever visited. But what is most remarkable about Rosetta's mission is what will happen when it reaches 67P/Churyumov-Gerasimenko in August 2014.

The spacecraft will be the first ever to orbit a comet, moving in as close as $20 \mathrm{~km}$ and analysing the surface of its nucleus to identify a suitable spot on which to land a probe. In November 2014, that probe, called Philae, will deploy and anchor itself to the nucleus, using an explosive harpoon. Philae will then image and analyse the changing composition of the comet as it makes its approach to the Sun, during 2015. The material from which $67 \mathrm{P} /$ Churyumov-Gerasimenko is formed is

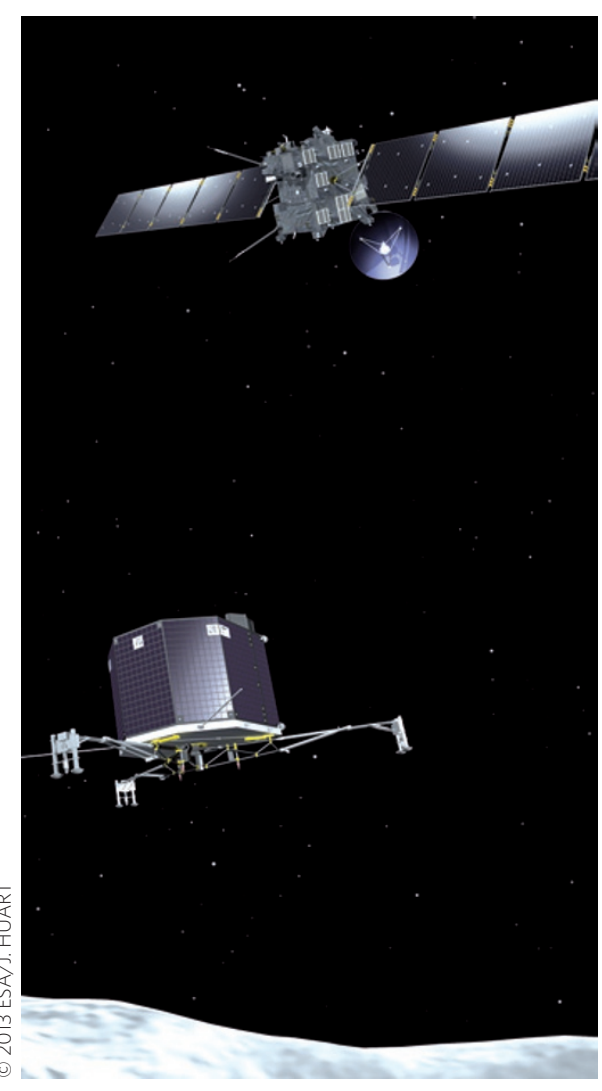

Rosetta and the lander Philae will reach their comet target later this year.

thought to be 4.6 billion years old, predating the Earth, and pristine - a brilliant window on the early Solar System.

There have been several successful missions to comets, although Rosetta is the most ambitious yet. In 1986, ESA's Giotto approached Halley's comet, and in 2006 NASA's Stardust probe returned a sample of the coma of comet Wild2. In 2005, another NASA mission, Deep Impact, launched an impactor into the nucleus of comet 9P/Tempel and photographed the resulting crater.

Asteroids are also useful targets. The mission of NASA's Dawn probe includes the two largest asteroids in the Solar System, the minor planet Vesta (orbited by Dawn in 2011) and the dwarf planet Ceres (which will be reached in 2015). In 2003, the Japanese space agency, JAXA, launched Hayabusa: the spacecraft made its rendezvous with the asteroid Itokawa in 2005, collected samples from the asteroid surface and returned them to Earth, landing in South Australia in 2010.

Interest in asteroids, of course, is not only about tracing the origins of the Solar System and understanding how the chemicals for life might have appeared on Earth. As with the Moon - which currently has China's Yutu rover trundling over its soil - asteroids are thought to offer new mining opportunities. Metals such as platinum, iridium and palladium could be extracted from near-Earth asteroids using low-cost spacecraft and certainly the success of exploratory missions such as Hayabusa, and the success so far of Rosetta, do not discourage the possibility.

But controversy developed last month after the publication of a report by Harvard astrophysicist Martin Elvis (Planet. Space Sci., in the press; preprint at http://arxiv.org/abs/1312.4450). Elvis' calculations suggest that the prospects for asteroid mining might not be quite so bright. There could be far fewer 'suitable' asteroids - close enough to Earth, big enough and rich enough in minerals than previously thought. The claim has been loudly criticized by companies who are exploring the possibility of asteroid mining, and Elvis himself is wary of the many uncertainties in the analysis. Inevitably, more work is needed to properly understand the true prospects and the likely return on investment, particularly if it becomes feasible to reach main-belt asteroids in orbit between Mars and Jupiter. It may also be a moment to consider the exploitation of such off-planet resources, and in parallel the depletion of the resources of this planet.

Curiously, the internet is a little vague on the exact origin of the 'string and sealing wax' phrase, although it seems likely to have evolved in the University of Cambridge's Cavendish Laboratory around the turn of the twentieth century, and might be due to J. J. Thomson. One entry has the full quote as, "If you can't do it with string and sealing wax on a lab bench, it probably isn't worth doing at all." Astronomers would no doubt disagree. 